\title{
Dual Graph Regularized NMF for Hyperspectral Unmixing
}

\author{
Lei Tong $^{1} \quad$ Jun Zhou $^{2} \quad \mathrm{Xiao} \mathrm{Bai}^{3} \quad$ Yongsheng Gao $^{1}$ \\ ${ }^{1}$ School of Engineering, Griffith University \\ Nathan, QLD 4111, Australia \\ ${ }^{2}$ School of Information and Communication Technology, Griffith University \\ Nathan, QLD 4111, Australia \\ ${ }^{3}$ School of Computer Science and Engineering Beihang University \\ Beijing 100191, China
}

\begin{abstract}
Hyperspectral unmixing is an important technique for estimating fraction of different land cover types from remote sensing imagery. In recent years, nonnegative matrix factorization $(N M F)$ with various constraints have been introduced into hyperspectral unmixing. Among these methods, graph based constraint has been proved to be useful in capturing the latent manifold structure of the hyperspectral data in feature space. In this paper, we propose to integrate graph-based constraint based on manifold assumption in feature space and consistency of data in spatial space to regularize the NMF method. Results on both synthetic and real data have validated the effectiveness of the proposed method.
\end{abstract}

\section{Introduction}

Hyperspectral remote sensing imagery, which contains both spatial and spectral information captured by spectral imaging sensors, has been widely used for ground information extraction [7]. Because of low spatial resolution of sensors, pixels in hyperspectral imagery are composed of mixed spectral responses from multiple ground objects. Therefore, hyperspectral unmixing has become a widely used method which decomposes mixed pixels into a collection of spectral signatures named endmembers and their corresponding proportions, i.e., abundance [14].

Linear mixture model treats each pixel as a linear combination of endmembers and assumes no interference between endmembers. A number of unmixing methods have been proposed based on linear mixture model $[4,13]$. Among them, there are two main unmixing categories: simplex geometric based or statistics based. The goal of simplex geometric based methods is to find the optimal vertices in the feature space to represent endmembers. N-FINDR [26] is one of the earliest approaches. The aim of this method is to find the maximum volume of simplex to fit the feature data and treat each vertex as an endmember. Pixel purity index (PPI) [5] generates some candidate endmembers via projection of the data. Then user is required to select endmembers from these candidates. Vertex component analysis (VCA) [21] projects the feature data to simplex and treats the vertices of the simplex as endmembers. A typical statistics based method is Independent Components Analysis (ICA) [8], which has been proposed as a means for both dimension reduction [24] and unmixing of hyperspectral data [20,3]. Sparse representation based approaches have also been reported $[11,1,10,9]$. They apply sparse constraints either to the endmember reconstruction step or to the abundances estimation step.

In recent years, nonnegative matrix factorization (NMF) [22] has been proposed as a statistics based unmixing method. It treats the unmixing as a blind source separation problem, and decomposes image data into two matrices, i.e., endmember matrix and abundance matrix, simultaneously. However, due to non-convexity of its object function, NMF algorithm usually fall into local minima. One solution is to add constraints to NMF according to different considerations. Miao and Qi [19] proposed a method named minimum volume constrained NMF which estimates endmembers outside of data that produce the minimum volume of formed simplex. Wang and Du [25] used the dissimilarity of the signatures as the constraint. Jia and Qian [12] forced the smoothness of both endmembers and abundance. Liu et al [17] proposed to use neighborhood information to enhance the unmixing performance by assigning weights to local neighborhood which is used as priori for the regularization term in the NMF model. Sparsity is an other important constraint in NMF method due to its physical interpretation. Qian et al proposed a sparsity constrained $L_{1 / 2^{-}}$ NMF method which allows each pixel be decomposed into 
only one or few endmembers [23].

One advantage of the sparsity constrained NMF method is that it can be combined with other constraints. For example, graph constraint based on manifold theory has been applied on top of $L_{1 / 2}$-NMF in the feature domain by Lu et al [18]. This method seeks the nearest neighbour of pixels in the feature space to build a graph that is used to guide the unmixing process. Closer pixels in the feature space therefore have similar unmixing results. In this paper, we propose to extend the graph approach from feature domain to spatial domain so that the spatial distances between pixels can be used as a novel graph constraint. This forces the consistency of unmixing among spatially close pixels. When such spatial constraint is combined with the feature space constraint, the derived dual graph constraint effectively models the relationship between pixels in both feature and spatial spaces. We show that the integration of this dual graph constraint and sparsity leads to more accurate unmixing results than several state-of-the-art approaches.

The rest of this paper is organised as follows. In Section 2, a brief overview of NMF unmixing background is given. Section 3 introduces the proposed unmixing method. Section 4 presents the experimental results. Finally, conclusions are drawn in Section 5.

\section{Background}

In hyperspectral image, each pixel can be considered as the mixture of several endmembers. Let the number of wavelength-indexed bands in an image be $H$ and the number of endmembers be $P$. A pixel $y$ in a hyperspectral image $Y$ is an $H \times 1$ column vector whose entries correspond to the reflectance of objects in different bands. Let $M$ be an $H \times P$ endmember matrix $\left(m_{1}, \ldots, m_{j}, \ldots, m_{P}\right)$, where $m_{j}$ is an $H \times 1$ column vector representing the spectral signature of the $j^{\text {th }}$ endmember. Then $y$ can be approximated by a linear combination of endmembers

$$
y=M r+e
$$

where $r$ is a $P \times 1$ column vector for endmember abundances, and $e$ is the additive Gaussian white noise.

Let $N$ be the number of pixels in $Y$, the linear mixture model of the whole image $Y$ can be defined as

$$
Y=M R+E
$$

where $R$ is a $P \times N$ abundance matrix which contains the proportion of each endmember at each pixel. $E$ is an $H \times N$ matrix representing the additive noise.

The goal of hyperspectral unmixing is to estimate the endmember matrix $M$ and the abundance matrix $R$ given a hyperspectral image $Y$. Apparently, both $M$ and $R$ shall be nonnegative because spectral responses of endmembers and their proportion at each pixel can not be smaller than zero. Because of this property, nonnegative matrix factorization (NMF) $[15,16]$, which decompose a matrix into nonnegative matrices, becomes a natural solution to hyperspectral unmixing.

\subsection{Sparsity Constrained NMF}

A disadvantage of NMF is that the cost function of NMF method is non-convex, so it does not lead to a unique solution. In order to solve this problem, $L_{1 / 2}$ sparsity constraint has been introduced [23], which leads to a sparse solution ( $L_{1 / 2}-\mathrm{NMF}$ ) of the abundance matrix. The sparsity of $R$ implies that only limited endmembers, but not all, will contribute to the formation of a pixel.

$L_{1 / 2}$-NMF defines the following objective function

$$
\operatorname{obj}(M, R)=\frac{1}{2}\|Y-M R\|_{F}^{2}+\lambda\|R\|_{1 / 2}
$$

where $\|\cdot\|_{F}$ represent the Frobenius norm, $\lambda$ weights the contribution of sparsity term $\|R\|_{1 / 2}$, and $\|R\|_{1 / 2}$ is define as

$$
\|R\|_{1 / 2}=\sum_{p=1}^{P} \sum_{n=1}^{N} R_{p n}^{\frac{1}{2}}
$$

A multiplicative iterative algorithm [16] is used to minimize the objective function, which is formulated as follows

$$
\begin{gathered}
M \leftarrow M . * Y R^{T} \cdot / M R R^{T} \\
R \leftarrow R . * M^{T} Y . /\left(M^{T} M R+\frac{\lambda}{2} R^{-\frac{1}{2}}\right)
\end{gathered}
$$

where $*$ and.$/$ are element-wise operations.

\section{Approach}

In this section, we first describe graph construction in both feature and spatial spaces. Then dual graph regularized NMF method is introduced. The proposed method also adopts $L_{1 / 2}$ sparsity to constrain the complexity of the estimated abundance matrix.

\subsection{Graph Construction}

Equation (1) tells that abundance vector $r$ can be considered as the projection of $y$ from an $H$ dimensional space to $P$ dimensional space, with $H \gg P$. Based on manifold learning theory [2], Lu et al [18] proposed that because hyperspectral image is high dimensional data, geodesics distance of the data manifold shall be considered to produce better hyperspectral unmixing performance. A good unmixing model shall preserve the manifold structure of the high dimensional space in the low dimensional space. Lu et al 
then proposed to build a nearest neighbor graph from high dimensional data by using the data points as vertices. The graph regularizer is incorporated into the NMF model so as to maintain consistent intrinsic structure of data in the feature space and the abundance matrix.

In more detail, the construction of this graph starts from searching for the k-nearest neighbours of each pixel in the high-dimensional data. If pixel $y_{i}$ is one of the k-nearest neighbors of pixel $y_{j}$, the weight is assigned as

$$
W_{1 i j}=\exp -\frac{\left\|y_{i}-y_{j}\right\|^{2}}{\sigma}
$$

which is the heat kernel. It is apparent that if the $y_{i}$ and $y_{j}$ are similar, the value of $W_{1 i j}$ is relatively large. An assumption is made here that when $y_{i}$ and $y_{j}$ are close in the feature space, their abundance $r_{i}$ and $r_{j}$ should also be close.

However, only using this manifold information may not be sufficient in fully characterizing the property of data in hyperspectral unmixing. In hyperspectral images, another important property is the spatial relationship between pixels. In particular, neighbouring pixels are likely to have similar spectral responses, and therefore, shall have similar unmixing results. Based on this consideration, we propose to build another graph in the spatial domain. In this spatial graph, the vertices are pixels, but the nearest neighbors are found based on spatial distance between pixels. Here, we use the first order neighbour as an example. The weight matrix of the graph is constructed as follows

$$
W_{2 i j}= \begin{cases}\frac{\pi}{2}-S A D\left(y_{i}, y_{j}\right), & y_{j} \in N\left(y_{i}\right) \text { or } y_{i} \in N\left(y_{j}\right) \\ 0, & \text { otherwise }\end{cases}
$$

where

$$
S A D\left(y_{i}, y_{j}\right)=\arccos \left(\frac{y_{i}^{T} y_{j}}{\left\|y_{i}\right\|\left\|y_{j}\right\|}\right)
$$

From this weight matrix, we can see if the signatures of neighbour pixels do not differ much, a large value will be assigned.

The above step generates two different graphs. One represents the high-dimensional data while the other deals with information of neighbourhood pixels. To regularize NMF method, we need to compute the Laplace matrix $L_{r}$ and diagonal matrix $D_{r}$ of $W_{r}$. Matrices $L_{r}$ and $D_{r}$ are computed as follows

$$
\begin{gathered}
D_{r i i}=\sum_{i=1}^{k} W_{r i j} \\
L_{r}=D_{r}-W_{r}
\end{gathered}
$$

Here, $r=1$ or $r=2$ indexes the graph defined in either the feature domain or the spatial domain, respectively. $k$ is the number of nearest neighbours, $i$ and $j$ are the indices of entries.

\subsection{Dual Graph Regularized NMF}

With two graphs being defined, the objective function of the regularized NMF can be defined as

$$
\begin{array}{r}
\operatorname{obj}(M, R)=\frac{1}{2}\|Y-M R\|_{F}^{2}+\lambda\|R\|_{1 / 2} \\
+\frac{\mu}{2} \alpha \operatorname{Tr}\left(R L_{1} R^{T}\right)+\frac{\mu}{2}(1-\alpha) \operatorname{Tr}\left(R L_{2} R^{T}\right)
\end{array}
$$

where $\mu \in R$ is a scalar that weights the contribution of graph constraints. $\operatorname{Tr}(\cdot)$ represents the trace of a matrix. As can be seen, the first two terms are the same as the formulation in the $L_{1 / 2}$-NMF method, which forces sparsity of the abundance matrix. The last two terms are the feature and spatial space regularization terms, respectively. $\alpha$ is a parameter to balance the contribution from the feature and spatial domain.

In order to get the optimal value of matrices $M$ and $R$, we need to take the partial derivative of equation (12) with respect to $M$ and $R$

$$
\begin{gathered}
\frac{\partial \operatorname{obj}(M, R)}{\partial M_{i j}}=-Y R^{T}+M R R^{T}+\Psi \\
\frac{\partial o b j(M, R)}{\partial R_{i j}}=-M^{T} Y+M^{T} M R+ \\
\frac{1}{2} \lambda R^{-\frac{1}{2}}+\mu \alpha R L_{1}+\mu(1-\alpha) R L_{2}+\Theta
\end{gathered}
$$

According to the Karush-Kuhn-Tucker conditions $\Psi_{h p} M_{h p}=0$ and $\Theta_{p n} R_{p n}=0$, we get

$$
\begin{gathered}
\left(-Y R^{T}+M R R^{T}\right) \cdot * M=0 \\
\left(-M^{T} Y+M^{T} M R+\frac{1}{2} \lambda R^{-\frac{1}{2}}+\right. \\
\left.\mu \alpha R L_{1}+\mu(1-\alpha) R L_{2}\right) \cdot * R=0
\end{gathered}
$$

By using transposition and division and $L_{r}=D_{r}-W_{r}$, the iterative updating rule of the dual graph regularized NMF becomes

$$
\begin{gathered}
M \leftarrow M . * Y R^{T} \cdot / M R R^{T} \\
R \leftarrow R . *\left(M^{T} Y+\mu R\left(\alpha W_{1}+(1-\alpha) W_{2}\right) \cdot /\right. \\
\left(M^{T} M R+\frac{\lambda}{2} R^{-\frac{1}{2}}+\mu R\left(\alpha D_{1}+(1-\alpha) D_{2}\right)\right)
\end{gathered}
$$

Note that the abundance matrix should also meet the sum-to-one constraint. In this paper, the data matrix $Y$ and the signature matrix $M$ are replaced by $Y_{f}$ and $M_{f}$ which are augmented matrices by a row of constants defined as follows

$$
Y_{f}=\left(\begin{array}{c}
Y \\
\delta 1_{N}^{T}
\end{array}\right) M_{f}=\left(\begin{array}{c}
M \\
\delta 1_{N}^{T}
\end{array}\right)
$$

where $\delta$ is a parameter to balance the impact of the additivity constraint on the abundance matrix. A large $\delta$ forces 
the sum of abundances at each pixel close to unity. In the iteration, these augmented matrices replace $Y$ and $M$ in the update rule of $R$ given in equation (18).

The procedure of our dual graph regularized NMF method is described below

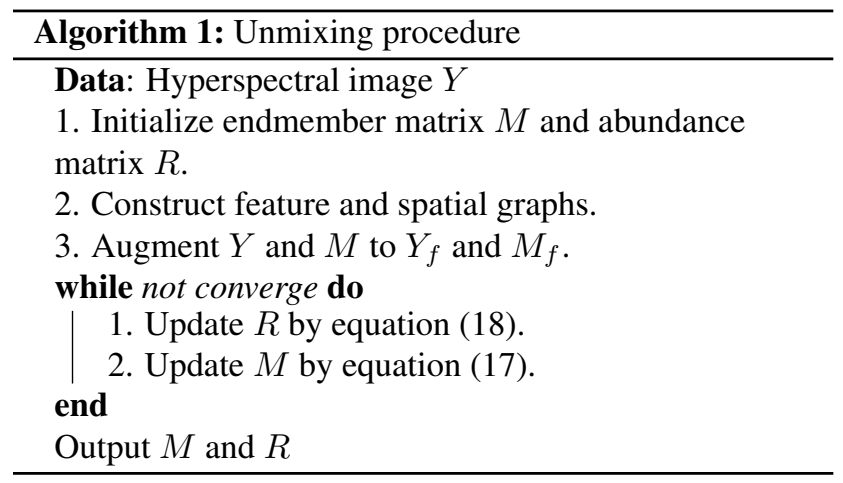

\section{Experiments}

A series of experiments are designed to evaluate the performance of the proposed dual graph regularized NMF method. Our method is compared with VCA [21], $L_{1 / 2}$-NMF [23], feature space graph regularized NMF (FGNMF) [18] and spatial graph regularized NMF (SGNMF) methods. In SGNMF method, the feature graph is replaced by a spatial graph generated in the same way as mentioned above.

In the experiments, we employ two metrics to evaluate the performance of these methods: Spectral Angle Distance (SAD) and Root Mean Square Error (RMSE). The SAD is used to compare the similarity of the $p^{t h}$ endmember signature $M_{p}$ and its estimate $\widehat{M}_{p}$, which is defined as

$$
\mathrm{SAD}_{p}=\arccos \left(\frac{M_{p}^{T} \widehat{M}_{p}}{\left\|M_{p}\right\|\left\|\widehat{M}_{p}\right\|}\right)
$$

On the other hand, the RMSE is used to evaluate the abundance estimates, which is defined as

$$
\operatorname{RMSE}_{p}=\left(\frac{1}{N}\left|R_{p}-\widehat{R}_{p}\right|^{2}\right)^{\frac{1}{2}}
$$

where $\widehat{R}_{p}$ is the ground-truth abundance matrix for the $p^{\text {th }}$ end-member while $R_{p}$ is the estimated one.

There are two stopping criteria in our method. The first is the maximum iteration number for the optimization step which is set to 3000 . The second is the error tolerance which is designed as

$$
\left|\mathcal{C}\left(M^{i}, R^{i}\right)-\mathcal{C}\left(M^{i-1}, R^{i-1}\right)\right| \leq \epsilon\left|\mathcal{C}\left(M^{i-1}, R^{i-1}\right)\right|
$$

where $\epsilon$ is set to $\epsilon=10^{-4}$ in our experiments. Once either of these criteria is met, the optimization process terminates. The value of the parameter $\lambda$ is dependent on the sparsity of the material abundances. Since these abundances cannot be obtained a priori, we use a rough estimator for $\lambda$ based on the sparseness criteria in [23].

\subsection{Synthetic Data}

We first evaluate and analyze the proposed method on synthetic data. Four spectral signatures are chosen from the USGS digital spectral library [6] to generate the synthetic data. Similar to [19], there are 5 steps to generate this synthetic data.

1. Generate an image which contains $64 \times 64$ pixels and divided into $8 \times 8$ regions.

2. In each region the same type of ground cover is initialized with one of the endmembers chosen randomly.

3. A low pass filter is applied to generate mixed data.

4. For the pixel whose abundance is larger than $\theta=0.8$, the abundance of this pixel is replaced with a mixture of all endmembers with equal abundances.

5. Zero-mean Gaussian noise is added to synthetic data.

The signal to noise ratio(SNR) is defined as

$$
S N R=10 \log _{10} \frac{E\left[y^{T} y\right]}{E\left[e^{T} e\right]}
$$

where the $y$ and $e$ are the observation and noise of a pixel. $E[\cdot]$ denotes the expectation operator.

We first evaluate the performance of different unmixing methods: VCA [21], $L_{1 / 2}$-NMF [23], FGNMF [18], SGNMF, and our method. In this experiment, the parameters setting in $L_{1 / 2}$-NMF, FGNMF, SGNMF, and our method are the same. We set $S N R=30$ and total number of endmember $P=4$ in this experiment. We run each method for 10 times and then calculate the mean value and standard deviation of SAD and RMSE. Fig. 1 shows the experimental results. The bars and error lines stand for the mean SADs and RMSEs and their standard deviations, respectively. It can be seen that our method has clearly outperformed all other methods in endmember estimation, and is marginally better than $L_{1 / 2}$-NMF and FGNMF methods in abundance estimation. In overall, our method is better than FGNMF and SGNMF because it uses both feature and spatial information while FGNMF and SGNMF only use one of them.

In the second experiment, different levels of SNR is tested to verify the robustness of our method. We set SNR as $15 \mathrm{~dB}, 25 \mathrm{~dB}, 35 \mathrm{~dB}, 45 \mathrm{~dB}$ and infinity (noise free) for 4 


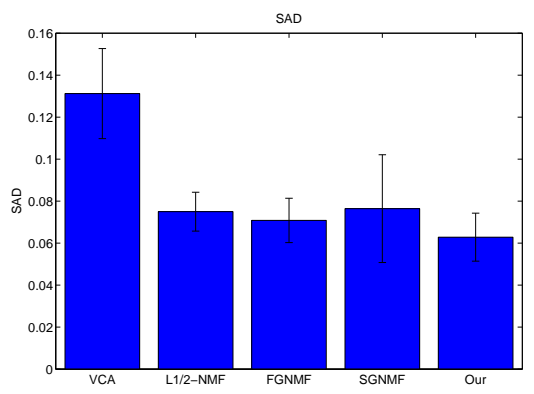

(a)

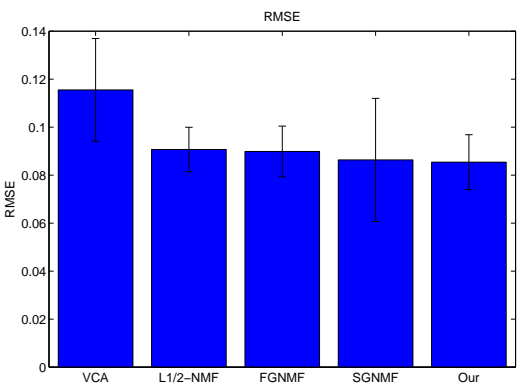

(b)

Figure 1. Performance of different algorithms. (a) Mean SAD; (b) Mean RMSE.

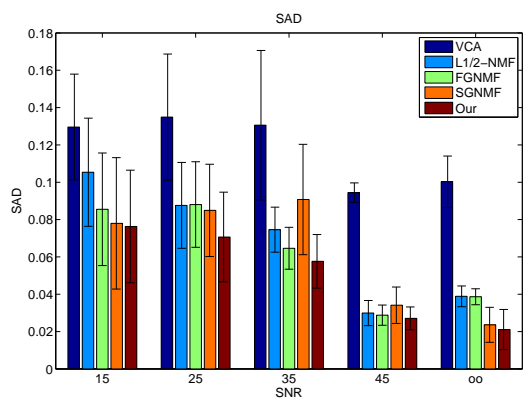

(a)

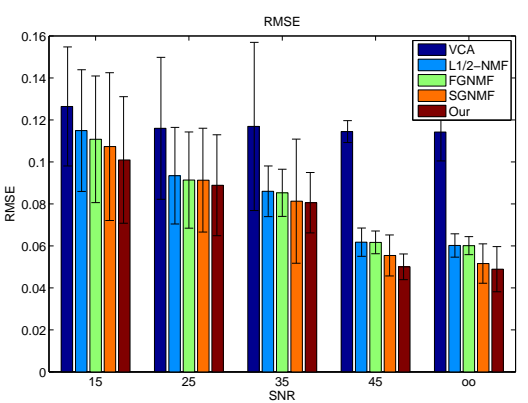

(b)

Figure 2. The result of different SNR. (a) Mean SAD; (b) Mean RMSE.

endmembers. Fig. 2(a) shows the SAD results on the endmember estimation from different methods. As we can see, our method outperforms all other methods. Meanwhile, Fig.2(b) shows that the RMSE of our method is also better than others no matter how the noise level changes. Our algorithm is clearly more robust to the influence from noise. This is due to the fact that it uses the intrinsic structure of hyperspectral data in both feature and spatial domain.

Robustness to different number of endmembers is another important aspect for unmixing method. In this experiment, we evaluate the performance of our method when the number of endmember changes. We set number of the endmembers from 4 to 8 , and $S N R=30 d B$. Fig. 3(a) shows the SAD of different method with number of endmembers in the synthetic data, while Fig. 3(b) shows the RMSE of the abundance estimation. From the figure we can see that no mater how many endmembers are there in the experiment, our method has better performance than all other methods.

\subsection{Remote Sensing Data}

We also conducted experiment on the widely used urban image obtained by the Urban Hyperspectral Digital Im-

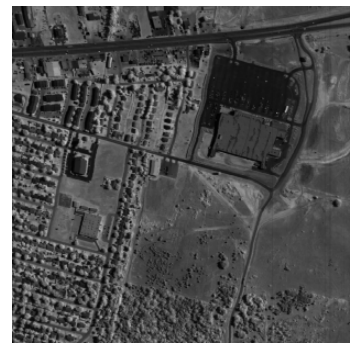
Figure 4. Urban HYDICE hyperspectral
dataset at band 80 .

agery Collection Experiment (HYDICE) sensor [23]. The image depicts the scene displayed in Figure 4 and is of size $307 \times 307$. This image is composed of 210 spectral channels with spectral resolution of $10 \mathrm{~nm}$ acquired in the $400 \mathrm{~nm}$ and $2500 \mathrm{~nm}$ region. After low SNR bands had been removed (channels 1-4, 76, 87, 101-111, 136-153, and 198-210), 162 bands remained for the experiments, i.e., $H=162$. 


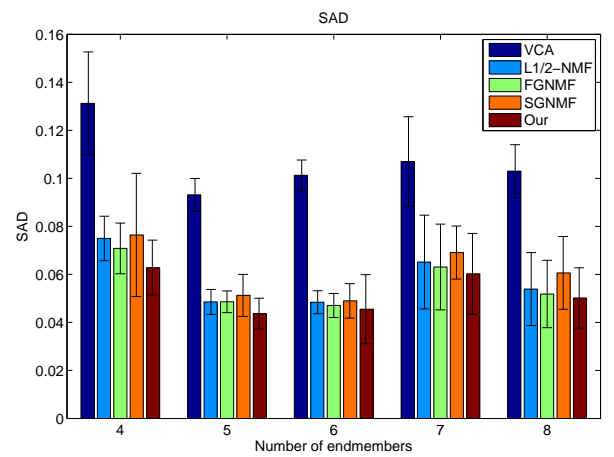

(a)

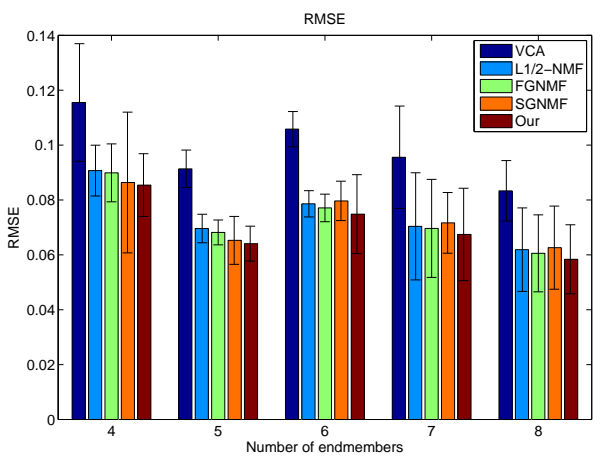

(b)

Figure 3. The result of increasing number of endmembers. (a) Mean SAD; (b) Mean RMSE.

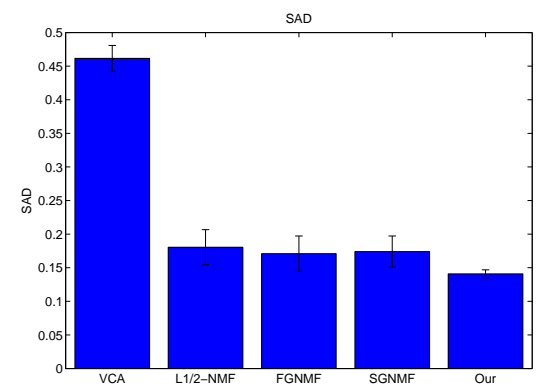

\section{Figure 5. Results of SAD on endmember esti- mation on the Urban HYDICE data.}

In the experiment, we considered 4 types of endmembers: Asphalt, Tree, Grass and Roof. Fig. 5 shows the mean SAD of endmember estimation with different methods. The results show that dual graph regularized NMF can help to get better endmember estimation. This confirms the effectiveness of combining both feature and spatial domain structural information for hyperspectral unmixing. Fig. 6 shows the abundance maps of endmembers. Each row represents abundance of an endmember. From top to bottom, they are the abundances of road, grass, asphalt and tree. Each column stands for different methods. From left to right, they are VCA, $L_{1 / 2}$-NMF, FGNMF, SGNMF and our method. From these abundance map, it can be seen that except the VCA method, the abundance maps of others are quite similar. Note that there is no ground truth on the abundance of each endmember, so it is difficult to give quantitative evaluation to the methods being compared.

\section{Conclusion}

In this paper, we have proposed a dual graph regularized NMF for hyperspectral unmixing. Built on top of the sparsity constrained NMF approach, this method provides new constraints on the NMF model by the construction of two graphs. The first graph leads to a regularization term based on the intrinsic manifold structure of data in the high dimensional feature space. The second graph is constructed to force the consistency of neighboring pixels in the unmixing results. An iterative optimization process is developed to generate a unified solution for both endmember and abundance estimation. This method is validated on both synthetic and real data, and have achieved better performance when compared against the single graph regularized NMF method. In the future, we will explore multiple graph regularized NMF for hyperspectral unmixing.

\section{References}

[1] N. Akhtar, F. Sahfait, and A. Mian. Repeated constrained sparse coding with partial dictionaries for hyperspectral unmixing. In IEEE Winter Conference on Applications of Computer Vision, pages 953-960, March 2014.

[2] M. Belkin and P. Niyogi. Laplacian eigenmaps and spectral techniques for embedding and clustering. In Advances in Neural Information Processing Systems, volume 14, pages 585-591, 2001.

[3] M. Berman, H. Kiiveri, R. Lagerstrom, A. Ernst, R. Dunne, and J. F. Huntington. ICE: A statistical approach to identifying endmembers in hyperspectral images. IEEE Transactions on Geoscience and Remote Sensing, 42(10):20852095, 2004.

[4] J. M. Bioucas-Dias, A. Plaza, N. Dobigeon, M. Parente, Q. Du, P. Gader, and J. Chanussot. Hyperspectral unmixing overview: Geometrical, statistical, and sparse regressionbased approaches. IEEE Journal of Selected Topics in Ap- 

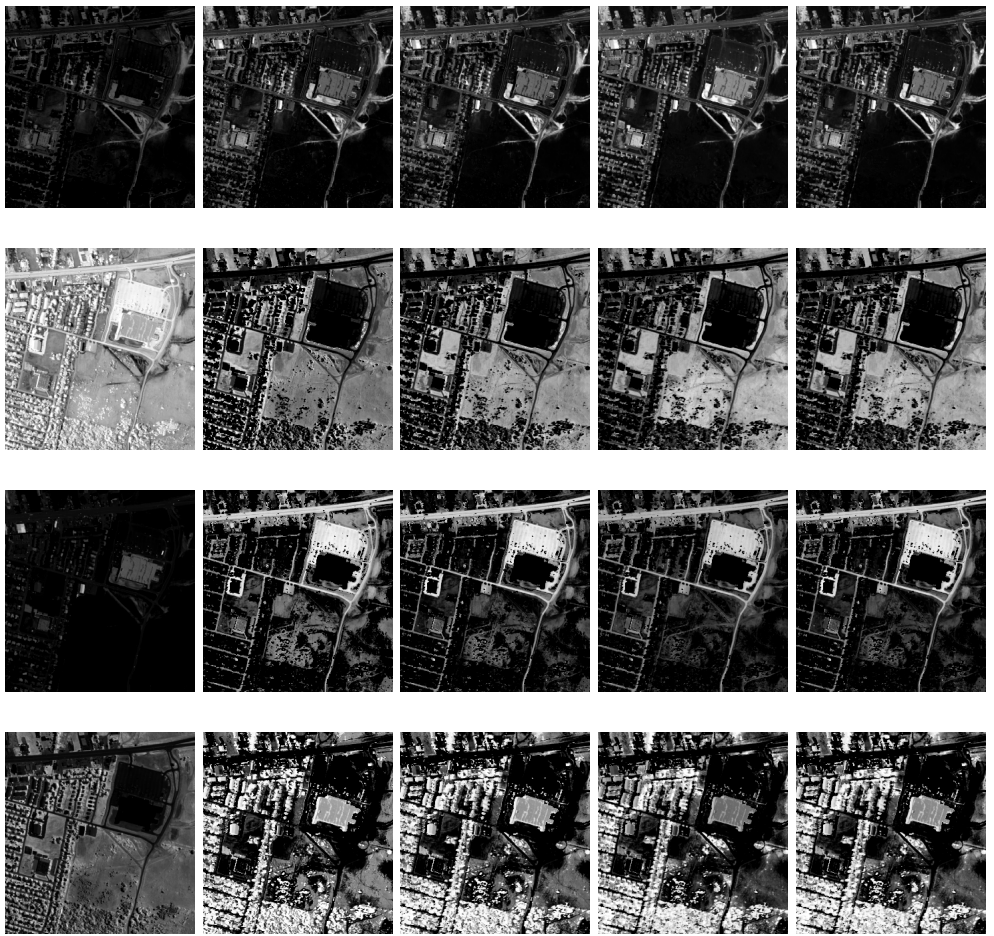

Figure 6. Results of abundance estimation on the Urban HYDICE data.

plied Earth Observations and Remote Sensing, 5(2):354379, 2012.

[5] J. W. Boardman. Automating spectral unmixing of aviris data using convex geometry concepts. In JPL Airborne Geoscience Workshop Summaries 4th Annu., volume 1, pages 11-14. JPL Publication 93-26, 1993.

[6] R. Clark, G. Swayze, R. Wise, E. Livo, T. Hoefen, R. Kokaly, and S. Sutley. USGS digital spectral library splib06a: U.S. Geological Survey, Digital Data Series 231. USGS, 2007.

[7] A. F. Goetz, G. Vane, J. E. Solomon, and B. N. Rock. Imaging spectrometry for earth remote sensing. Science, 228(4704):1147-1153, 1985.

[8] A. Hyvärinen and E. Oja. Independent component analysis: algorithms and applications. Neural networks, 13(4):411430, 2000.

[9] M. Iordache, J. Dias, and A. Plaza. Total variation spatial regularization for sparse hyperspectral unmixing. IEEE Transactions on Geoscience and Remote Sensing, 50(11):4484-4502, December 2012.

[10] M. Iordache, J. Dias, and A. Plaza. Collaborative sparse regression for hyperspectral unmixing. IEEE Transactions on Geoscience and Remote Sensing, 52(1):341-354, January 2013.

[11] M.-D. Iordache, J. Bioucas-Dias, and A. Plaza. Sparse unmixing of hyperspectral data. IEEE Transactions on Geoscience and Remote Sensing, 49(6):2014-2039, June 2011.

[12] S. Jia and Y. Qian. Constrained nonnegative matrix factorization for hyperspectral unmixing. IEEE Transactions on Geoscience and Remote Sensing, 47(1):161-173, 2009.
[13] N. Keshava. A survey of spectral unmixing algorithms. Lincoln Laboratory Journal, 14(1):55-78, 2003.

[14] N. Keshava and J. F. Mustard. Spectral unmixing. IEEE Signal Processing Magazine, 19(1):44-57, 2002.

[15] D. D. Lee and H. S. Seung. Learning the parts of objects by non-negative matrix factorization. Nature, 401(6755):788$791,1999$.

[16] D. D. Lee and H. S. Seung. Algorithms for non-negative matrix factorization. In Advances in neural information processing systems, pages 556-562, 2000.

[17] J. Liu, J. Zhang, Y. Gao, C. Zhang, and Z. Li. Enhancing spectral unmixing by local neighborhood weights. IEEE Journal of Selected Topics in Applied Earth Observations and Remote Sensing, 5(5):1545-1552, 2012.

[18] X. Lu, H. Wu, Y. Yuan, P. Yan, and X. Li. Manifold regularized sparse NMF for hyperspectral unmixing. IEEE Transactions on Geoscience and Remote Sensing, 51(5):28152826, 2013.

[19] L. Miao and H. Qi. Endmember extraction from highly mixed data using minimum volume constrained nonnegative matrix factorization. IEEE Transactions on Geoscience and Remote Sensing, 45(3):765-777, 2007.

[20] J. M. Nascimento and J. M. Bioucas Dias. Does independent component analysis play a role in unmixing hyperspectral data? IEEE Transactions on Geoscience and Remote Sensing, 43(1):175-187, 2005.

[21] J. M. Nascimento and J. M. Dias. Vertex component analysis: A fast algorithm to unmix hyperspectral data. IEEE Transactions on Geoscience and Remote Sensing, 43(4):898-910, 2005. 
[22] V. P. Pauca, J. Piper, and R. J. Plemmons. Nonnegative matrix factorization for spectral data analysis. Linear algebra and its applications, 416(1):29-47, 2006.

[23] Y. Qian, S. Jia, J. Zhou, and A. Robles-Kelly. Hyperspectral unmixing via $L_{1 / 2}$ sparsity-constrained nonnegative matrix factorization. IEEE Transactions on Geoscience and Remote Sensing, 49(11):4282-4297, 2011.

[24] J. Wang and C.-I. Chang. Independent component analysisbased dimensionality reduction with applications in hyperspectral image analysis. IEEE Transactions on Geoscience and Remote Sensing, 44(6):1586-1600, 2006.

[25] N. Wang, B. Du, and L. Zhang. An endmember dissimilarity constrained non-negative matrix factorization method for hyperspectral unmixing. IEEE Journal of Selected Topics in Applied Earth Observations and Remote Sensing, 6(2):554569, April 2013.

[26] M. E. Winter. N-FINDR: an algorithm for fast autonomous spectral end-member determination in hyperspectral data. In SPIE International Symposium on Optical Science, Engineering, and Instrumentation, pages 266-275. International Society for Optics and Photonics, 1999. 